\title{
Mas allá del supuesto de continuidad. Minimalismo radical en niños con TEL
}

\author{
Beyond the continuity assumption. Radical \\ minimalism in children with SLI
}

\author{
GUILLERMO LORENZO \\ Universidad de Oviedo \\ Elena VARes \\ Escuelas Universitarias Gimbernat-Cantabria
}

\begin{abstract}
Resumen. Este trabajo se plantea la cuestión de si las formas de lenguaje a que dan lugar los trastornos del desarrollo son continuas en relación con las formas propias del desarrollo típico. Se sugiere, en concreto, la adopción de una perspectiva que, paralelamente a la teoría generativa contemporánea, va "más allá" del criterio de adecuación explicativa y aborda el caso desde la perspectiva del llamado "problema de diseño". El planteamiento implica preguntarse si el lenguaje desarrollado atípicamente obedece al tipo de criterios de optimización de diseño que hacen del lenguaje, técnicamente hablando, un objeto "cuasi" perfecto. El trabajo se basa en el análisis de una muestra de español de niños con TEL obtenida experimentalmente, que nos lleva a concluir que, en efecto, en las gramáticas subyacentes prevalecen los efectos de optimización (o "tercer factor") sobre los principios gramaticales específicos de dominio.
\end{abstract}

Palabras clave: Trastorno específico del lenguaje, Programa minimalista, Gramática, Tercer factor, Supuesto de continuidad

Abstract. Parallel to classical debates in the field of language acquisition, the question can be
raised whether developmental language disorders are continuous in comparison to developmentally

Data de recepción: 08-08-2018 - Data de aceptación: 20-11-2018.

I VERBA, ISSN 0210-377X, 2019, vol. 46: 371-401 - SECCIÓN: ARTIGOS - http://dx.doi.org/10.15304/verba.46.5405 I 
typical language. Matters are contentious, but what we suggest in this paper is a new perspective on the issue, namely, moving 'beyond' explanatory adequacy and dealing with the case from the standpoint of the 'problem of design.' This point of view leads us to the question whether developmentally atypical language still obeys the kinds of design optimization criteria that make language a quasi-perfect object. The analysis of a sample of experimentally gathered child SLI Spanish leads us to conclude that, indeed, optimization/third factor effects prevail over UG principles in their grammars.

Keywords: Specific Language Impairment; Minimalist Program; Universal Grammar; Third Factor; Continuity Assumption

\section{INTRODUCCIÓN}

En 1984, Steven Pinker planteó que, como un caso particular de la sugerencia más general formulada por Macnamara (1982), sería razonable asumir, en ausencia de pruebas refutatorias claras, que las gramáticas infantiles consisten en los mismos materiales (unidades, reglas, principios, etc.) que las gramáticas de los adultos. En concreto, Pinker planteó que, de este modo, "en la situación ideal, solo tendríamos que atribuir al niño mecanismos cognitivos extralingüísticos que ya tienen motivación independiente en el estudio de la cognición del adulto", añadiendo que "esta asunción debería aplicarse no únicamente a los mecanismos cognitivos del niño, sino también a sus mecanismos gramaticales" (Pinker 1984: 7; traducción de los autores). El "supuesto de continuidad" resultante lo formuló el propio Pinker en los siguientes términos:

(1) Supuesto de continuidad (SC)

En ausencia de evidencia convincente en sentido contrario, las reglas gramaticales del niño deben consistir en el mismo tipo básico de reglas y constar de símbolos primitivos de la misma clase que las reglas gramaticales atribuidas a los adultos en la investigación estándar (Pinker 1984: 7; traducción de los autores).

A primera vista, el SC aparenta ser una posición "adulto-céntrica", en línea con el tipo de asunciones que la psicobiología del desarrollo sugiere evitar por entrar en conflicto con la premisa básica de que ninguna capacidad orgánica puede dejar de tener un historial de maduración (Michel y Moore 1995: 22). Ciertamente, el propio Pinker parece ir a contracorriente en este sentido si tenemos en cuenta la continuación de la cita anterior, en la que añade que, si se acepta el SC, "el peso de la prueba recae en los defensores del cambio maduracional" (Pinker 1984: 7; traducción de los autores). No está claro, sin embargo, que la tesis de Pinker sea necesariamente 
reflejo de una posición adulto-céntrica, ya que el SC no es incompatible con un tipo de transformación cuantitativa de la gramática infantil en adulta, sin que tenga lugar la ruptura de una tipología común de reglas y símbolos. De hecho, la posición de Pinker también podría tildarse de "infanto-céntrica", puesto que el supuesto es perfectamente reversible en lo que se refiere a su posible formulación. Pinker argumenta que resulta relativamente más forzada, y por tanto no debería funcionar como hipótesis por defecto, la idea alternativa. Según esta, los niños siguen un camino discontinuo al adquirir una lengua, a través de estadios sucesivos consistentes en tipos de reglas y unidades de un género cualitativamente diferente, como en la llamada hipótesis "del huevo al renacuajo a la rana" (Slobin 1980; Gleitman 1981). Desde la publicación de todas estas referencias clásicas, el SC ha sido objeto de constante controversia entre los adquisicionistas (véanse Tomasello 2000 y Crain 2002 como referencias representativas, respectivamente, de las posturas en contra y a favor). En este trabajo, nos sumamos a la metodología de Pinker, aunque admitiendo el carácter en último término empírico de la cuestión.

La aportación empírica de este trabajo se centra en casos de adquisición atípica del lenguaje, que dan lugar a formas de trastorno específico del lenguaje (TEL). El término TEL se aplica a las afectaciones en la pauta de desarrollo y competencia alcanzada en diversas áreas del lenguaje, particularmente la morfosintaxis, en ausencia de otras condiciones patológicas en la psicomotricidad o la cognición, en un sentido más amplio, que pudieran explicarlas (véase, por ejemplo, Mendoza Lara 2016; Sanz Torrent y Serra 2017; Vares 2018). Los datos proceden de hablantes nativos de español (edad: 5;1 a 15;00), todos los cuales tienen un diagnóstico oficial de TEL, como se especificará a continuación con más detalle. El SC se aplica a estos casos si los interpretamos como ralentizaciones con relación al cronograma típico de adquisición (Rice et al. 1995), o como reflejo de afectaciones muy concretas (Clahsen 1989; Gopnik y Crago 1991; Rice y Wexler 1996; van der Lely 1998), aunque respetando los mismos principios o tipos de reglas y unidades que se aplican en la adquisición y estructuran el lenguaje adquirido típicamente. Leonard (1989) representa un temprano ejemplo de adhesión a esta postura continuista. Otros autores consideran, en cambio, que los niños con TEL manifiestan una carencia generalizada de recursos representacionales propios de las gramáticas naturales, lo que les impide solucionar con eficacia los problemas de aprendibilidad propios de la adquisición temprana. En consecuencia, tanto sus trayectorias de desarrollo como sus gramáticas son "desviadas" respecto a los correlatos típicos (Penner et al. 1999; Penner et al. 2003).

En todo caso, nuestro principal objetivo con este trabajo consiste en avanzar hacia una reformulación del debate, situándolo en un nivel de teorización en que, a 
lo que alcanza nuestro conocimiento, no ha sido anteriormente tratado. En concreto, el SC ha sido habitualmente considerado como un aspecto controvertido de la "adecuación explicativa" de la teoría lingüística, es decir, su capacidad para explicar los principales hitos de la adquisición, teniendo en cuenta que el proceso tiene lugar en un contexto de estimulación en varios sentidos "pobre". Es decir, muchas reglas no están representadas, o al menos no abundantemente, en los modelos ambientales al alcance del niño; el estímulo, que es puramente lineal, no transparenta sus principios organizativos básicos, que son estructurales; la cantidad de estímulo puede ser, en algunos casos, cuantitativa o cualitativamente deficiente (véanse Berwick et al. 2011, y Chomsky 2016, para una actualización de la idea). Nosotros sugerimos, en cambio, que el debate puede también plantearse "más allá" de tal nivel de adecuación, adoptando la terminología de Chomsky (2004) para definir los objetivos del Programa minimalista (PM). La cuestión pasará a girar entonces en torno a interrogantes como los siguientes: ¿son conformes las gramáticas infantiles a los mismos criterios de optimización que supuestamente constriñen el diseño de las gramáticas adultas? Y, ya más cerca de nuestros propios intereses, ¿son conformes las gramáticas adquiridas atípicamente a esos mismos criterios? En las siguientes páginas aportamos algunas pruebas de que parece ser efectivamente así y que, al menos en algunos dominios de gramaticalidad, incluso de una forma más acusada. Llamamos al fenómeno "minimalismo radical". Con el término pretendemos expresar que efectos de "tercer factor" (Chomsky 2005) parecen asumir en estos casos tareas típicamente propias de principios gramaticales estándar, lo que los aproxima así más al ideal chomskiano de "perfección" lingüística. Tales aportaciones al diseño del lenguaje, que Chomsky suma a la contribución del genotipo lingüístico ("primer factor") y de los modelos ambientales ("segundo factor"), consisten en principios de alcance muy general (no específicos del lenguaje, por tanto) relacionados con leyes generales de la forma, el acomodo a principios globales del procesamiento de datos, y a pautas o constricciones ineludibles del desarrollo (Chomsky 2005: 6). Planteamos, por tanto, que los niños con TEL son "minimalistas radicales", en este preciso sentido, en algunas áreas de su desempeño lingüístico.

El trabajo está organizado del siguiente modo. Las secciones 2 y 3 aportan los datos en que basamos la hipótesis anterior, tomados de dos áreas en particular de la gramática del español: la composición en términos de rasgos de los clíticos pronominales (sección 2) y ciertos efectos sui generis de "arrastre" observados en la formación de preguntas (sección 3). A partir de tales datos, en la sección 4 elaboramos nuestra tesis de que el SC tiene aplicación en diferentes niveles de profundidad teórica, incluyendo el nivel que Chomsky (2004) localiza "más allá de la adecuación explicativa", de 
forma particularmente significativa en los casos de desarrollo atípico. La sección 5 es un apartado de conclusiones.

\section{PRUEBAS DE MINIMALISMO RADICAL: CONCORDANCIA DE SUJETO EN CLÍTICOS DE OBJETO}

En esta sección presentamos algunos resultados de una investigación sobre la producción de oraciones con clíticos pronominales de objeto de una población de niños hispanohablantes con TEL, en la que destaca la proliferación de formas en que la morfología de concordancia se corresponde con la propia del sujeto. La sección 2.1 explica la procedencia de nuestras primeras constataciones del fenómeno e introduce el paradigma experimental posteriormente aplicado para confirmar su prevalencia en la población estudiada. También presenta las características de dicha población y los resultados obtenidos. En la sección 2.2 se interpretan, concluyendo que se deben a la aplicación de un principio básico de economía, en ausencia del condicionante gramatical que actúa en las gramáticas típicas.

\subsection{Participantes, materiales y resultados}

Existe una extensa bibliografía sobre el TEL en hablantes monolingües o bilingües con el español como una de sus lenguas en que se apunta genéricamente a la alta prevalencia de dificultades con los pronombres clíticos, superior, por ejemplo, a las observadas en la flexión verbal (Restrepo y Kruth 2000; Restrepo et al. 2004; Paradis et al. 2006; Simon-Cereijido y Gutiérrez-Clellen 2007; Morgan et al. 2009). Los estudios más detallados sobre la cuestión observan que son comunes los casos de omisión o sustitución, especialmente cuando se corresponden con plurales, y señalan la especial incidencia de los errores en la concordancia de género (Anderson 2001, 2003; Gutiérrez-Clellen y Peña 2001; Jacobson y Schwraz 2002; Bedore y Leonard 2005; Gutiérrez-Clellen et al. 2006). En nuestros propios test orientados a evaluar genéricamente la producción de clíticos pronominales, encontramos de forma inesperada una tendencia a producir clíticos de objeto con morfología de sujeto. Nuestros test iniciales (Experimento 1) se basaban en la presentación de fotografías acompañadas de oraciones. Los niños eran entrenados para reemplazar el SN objeto de la oración por un pronombre seleccionado entre cuatro posibilidades (Figura 1).

Cada uno de los ítems en que consistía el test era presentado en una diapositiva independiente, que contenía inicialmente una imagen y una oración. Al mismo tiempo, el examinador leía la oración en voz alta. Las oraciones eran en todos los casos del 




FIGURA 1. Sustitución de un SN (la guitarra) por un clítico pronominal de objeto $(l a)$. Opciones inválidas: lo, los, las.

tipo “sujeto-verbo-objeto". Una vez leída, el objeto de la oración era tachado, y en ese momento se pedía al niño que escogiese una de entre cuatro opciones que entonces aparecían en la diapositiva, en cada una de las cuales un pronombre diferente sustituía al SN objeto original. Estas opciones también eran leídas en voz alta por el examinador. Los clíticos pronominales pertenecían en todos los casos al paradigma del acusativo $(l o, l a, l o s, l a s)$. En las oraciones había tanto muestras de enclisis (con verbos en infinitivo, gerundio o imperativo) como de proclisis (con verbos finitos), distribuidas de igual modo y en idéntico número. El examinador repetía la primera oración y las subsiguientes opciones tantas veces como lo requerían los niños, a quienes se pedía que leyesen en voz alta la opción elegida. Los niños participaron en ensayos con ítems semejantes a los de los test definitivos, que solo comenzaban cuando el examinador estaba seguro de que habían entendido su lógica.

Estos primeros test fueron realizados por 16 niños con TEL (edad: 6;0 a 12;0, media: 8;9) y por 16 niños con desarrollo lingüístico típico, que sirvieron como controles. Cada niño realizó un test consistente en un total de 40 ítems. El contacto con los niños con TEL fue realizado a través de asociaciones de familias con afectados. Estos niños estaban integrados en el sistema escolar estándar, recibiendo refuerzo profesional bien en el colegio bien en otras instituciones. Los niños con TEL contaban con un diagnóstico oficial, puesto a disposición de los investigadores por sus familiares. Todos habían sido sometidos por profesionales a test homologados que descartaban síntomas de espectro autista (ADI-R y ADOS-G) o dislexia (pruebas de desarrollo cognitivo y motor conformes a la edad), y mostraban que se encontraban en rangos típicos de inteligencia no verbal (Leiter, TONI, WISC no verbal). Los diagnósticos incluían anamnesis del entorno familiar que excluían trastornos auditivos o problemas físicos 
de otro tipo ${ }^{1}$. Nuestras propias pruebas mostraron que sus dificultades con la gramática se extendían mucho más allá de los aspectos considerados en los test. Los controles, equiparados en edad con los sujetos experimentales, fueron seleccionados atendiendo a la similitud de sus entornos escolares y socio-económicos. En el momento en que fueron realizados los experimentos, nuestra institución académica no tenía establecido un protocolo ético para este tipo de investigación. Los test fueron de todos modos realizados con los consentimientos oportunos. La Tabla 1 ofrece el detalle de nuestros hallazgos en el Experimento 1 y la Figura 2 los visualiza gráficamente.

\begin{tabular}{|l|c|c|c|c|c|c|}
\hline & \multicolumn{4}{|c|}{ Grupo TEL } & \multicolumn{3}{c|}{ Grupo Control } \\
\hline & Enclisis & Proclisis & Total & Enclisis & Proclisis & Total \\
\hline Errores de género & 60 & 70 & $130 / 640(20,3 \%)$ & 2 & 4 & $6 / 640(0,9 \%)$ \\
\hline Errores de número $^{\mathrm{b}}$ & 53 & 40 & $93 / 640(14,5 \%)$ & 4 & 1 & $5 / 640(0,8 \%)$ \\
\hline E. género y número $^{\mathrm{c}}$ & 36 & 28 & $64 / 640(10 \%)$ & 1 & 0 & $1 / 640(0,2 \%)$ \\
\hline Total & 149 & 138 & $287 / 640(44,8 \%)$ & 7 & 5 & $12 / 640(1,9 \%)$ \\
\hline Concordancia con sujeto & 63 & 56 & $119 / 413^{1}(28,8 \%)$ & 5 & 1 & $6 / 413^{1}(1,4 \%)$ \\
\hline $\begin{array}{l}\text { 1. Posibles contextos para la concordancia con el sujeto. En los restantes contextos, la coincidencia en rasgos del } \\
\text { sujeto y el objeto dejan indeterminada la orientación de la concordancia, por lo que no son computados. }\end{array}$ \\
$\begin{array}{l}\text { a. Yo suelo desayunar cereales } \rightarrow \text { Yo suelo desayunarlas (LF 9;7) } \\
\text { b. El albañil construye la casa } \rightarrow \text { El albañil las construye (NX 10;8) } \\
\text { c. El fantasma asustó a Ana } \rightarrow \text { El fantasma los asustó (CR 8;10) }\end{array}$ \\
\hline
\end{tabular}

TABLA 1. Detalles generales de las producciones de los grupos TEL y control en el Experimento 1.

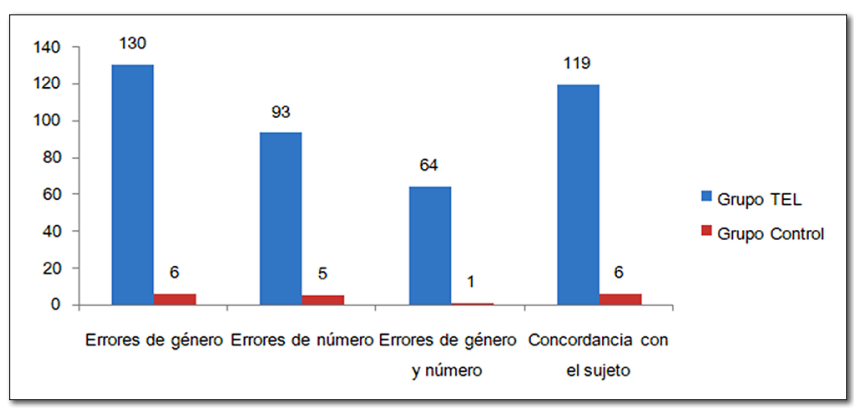

FIGURA 2. Visualización gráfica de los detalles de la Tabla 1.

Agradecemos a DELETREA, Centro de Psicología y Lenguaje (Madrid), por su asesoramiento acerca de los protocolos diagnósticos para la identificación del TEL, que nos ha permitido contrastar la validez de los puestos a nuestra disposición por los tutores de los participantes en las pruebas. 
Una vez identificada la tendencia de los clíticos de objeto a concordar con el sujeto, llevamos a cabo una segunda serie de test (Experimento 2) orientada a confirmar tal sesgo, para la que fueron incorporados más sujetos experimentales y se amplió la edad mínima de estos. En esta ocasión, los objetos fueron escogidos de modo que se diferenciasen en todos los casos del sujeto en género y número. A los niños se les ofrecía una imagen acompañada de sólo dos opciones entre las que elegir, una en la que el clítico se correspondía en rasgos con el sujeto y otra en que lo hacía con el objeto (Figura 3). Previamente, se les ofrecieron dos muestras y el examinador repitió el funcionamiento del test tantas veces como se le requirió.



FIgURA 3. Sustitución de un SN (la televisión) por un clítico pronominal de objeto (la). Opción inválida: los.

Estos test confirmaron la tendencia a hacer concordar el clítico con el sujeto, puesto que los errores se incrementaron cuando los ítems experimentales solo mostraban oraciones en las que el sujeto y el objeto diferían sistemáticamente en su composición en términos de género y número. En el Experimento 1, los niños con TEL usaron concordancia de objeto el 28,8\% de las ocasiones; en el Experimento 2, la cifra alcanzó el 45,1\% (Tabla 2), un porcentaje estadísticamente significativo relativamente a los controles $(Z=-11,288 ; \mathrm{p}=.000)$. En este segundo experimento, decidimos incrementar el número de niños (23) y el rango de edad (5;1 a 15;0, media: $10 ; 4)$, en todos los casos con niños con perfiles muy similares a los de los test originales, después de verificar que las pruebas eran comprensibles por parte de los niños de menor edad. No observamos ninguna tendencia significativa: los errores se encontraban igualmente distribuidos a lo largo de todo el rango de edades, sin que se observase una mayor incidencia en los niños de menor edad (Tabla 3). La inclusión de estos niños nos permitió así reafirmar la fuerza de la generalización planteada. 
Siguen algunos ejemplos ilustrativos obtenidos en el Experimento 2, antecedidos por las respuestas esperables (y, entre paréntesis, el objeto inicialmente presente y luego elidido en las dispositivas):

(2) a. Los chicos la ven (la televisión)

b. Los chicos los ven (C 5;1)

(3) a. Las chicas los limpian (los libros)

b. Las chicas las limpian (L 8;9)

(4) a. La chica las busca (las llaves)

b. La chica la busca $(\mathrm{U} 11 ; 0)$

(5) a. La chica lo come (un pastel)

b. La chica la come (V 5;7)

(6) a. El niño la pone (la chaqueta)

b. El niño lo pone (DV 7;4)

(7) a. Las chicas la beben (la leche)

b. Las chicas las beben (DG 8;0)

\begin{tabular}{|c|c|c|}
\hline \multirow{2}{*}{} & \multicolumn{2}{|c|}{ Concordancia de sujeto } \\
\cline { 2 - 3 } & Grupo TEL & Grupo control \\
\hline Experimento 1 & $28,8 \%$ & $1,4 \%$ \\
\hline Experimento 2 & $45,1 \%$ & $3,53 \%$ \\
\hline
\end{tabular}

TABLA 2. Resultados de los experimentos orientados a evaluar el uso de los clíticos pronominales de objeto. Usos con concordancia de sujeto inesperada.

\begin{tabular}{|c|c|c|c|c|c|}
\hline & $\mathbf{5 ; 1 - 7 ; 4}$ & $\mathbf{7 ; 5 - 9 ; 1 1}$ & $\mathbf{1 0 ; 0 - 1 1 ; 2}$ & $\mathbf{1 1 ; 3 - 1 3 ; 7}$ & $\mathbf{1 3 ; 8 - 1 5 ; 0}$ \\
\hline \multirow{2}{*}{ Grupo TEL } & $43 / 368$ & $36 / 368$ & $36 / 368$ & $10 / 368$ & $41 / 368$ \\
\cline { 2 - 6 } & $(11,7 \%)$ & $(9,8 \%)$ & $(9,8 \%)$ & $(2,7 \%)^{1}$ & $(11,1 \%)$ \\
\hline \multirow{2}{*}{ Grupo control } & $11 / 368$ & $2 / 368$ & 0 & 0 & 0 \\
\cline { 2 - 6 } & $(2,9 \%)$ & $(0,54 \%)$ & & & \\
\hline
\end{tabular}

1. Este valor se debe al menor número de informantes en esta franja. La media de errores (8) es, sin embargo, superior a la de la siguiente franja de edad $(6,2)$.

TABLA 3. Desglose de los errores registrados en la Tabla 2 correspondientes al Experimento 2, distribuidos por el rango de edades. 


\subsection{Análisis y discusión}

Sugerimos un análisis de los datos destacados en la subsección anterior basado en el modelo "sonda-meta" de Chomsky (2001), de acuerdo con el cual:

1. los rasgos de persona, género y número de los clíticos pronominales son "no interpretables" (es decir, réplica, en condiciones normales, de los del objeto) y entran en la composición de la oración pendientes de ser "validados"; en consecuencia,

2. necesitan "buscar" el correlato interpretable más próximo en su dominio capaz de validarlos; de este modo,

3. se produce una relación de "concordancia" que permite la "transferencia" de los rasgos relevantes; y así,

4. los clíticos pueden ser procesados como unidades interpretables válidas a efectos del pensamiento expresado.

El carácter "no interpretable" que atribuimos en el punto 1 de esta enumeración a los clíticos se hace particularmente manifiesto en las construcciones con "doblado" (por ejemplo, esa canción ya la toqué). En los casos en que el clítico y el objeto léxico se distribuyen complementariamente (por ejemplo, los chicos ven la televisión o los chicos la ven), entendemos que los clíticos buscan los rasgos de un objeto pronominal abstracto ('pro'; por ejemplo, los chicos la ven pro $_{3 \mathrm{p} \text {-fem-sing }}$ ), aptos para validarlos.

Nos servimos, además, de la estructura de frase sugerida por Chomsky para las construcciones transitivas, consistente en una capa superior $\mathrm{S} v$, con un núcleo $v$ (una especie de verbo abstracto que aglutina la expresión de las nociones de "agentividad" y "transitividad"), un SN sujeto en la posición de especificador, y un SV en la de complemento, cuyo núcleo expresa el tipo de acción verbal y cuyo complemento, en los casos que aquí nos interesan, es el pronombre abstracto con los rasgos interpretables correspondientes. La razón de ser del nivel de estructura $\mathrm{S} v$ guarda relación con la llamada "Generalización de Burzio" (Burzio 1986), que capta la conexión entre el hecho de que las oraciones que contienen un SN desempeñando el papel semántico de agente, portador del rasgo "nominativo", sean consecuentemente transitivas y contengan un objeto portando el rasgo "acusativo". El clítico ocupa, de acuerdo con nuestro análisis, una posición por encima del $\mathrm{S} v$, por tanto, también superior al sujeto. De acuerdo con la sugerencia de un revisor anónimo, se trataría de una de las posiciones del área clítica que introducen estos elementos en las lenguas romances, en consonancia con el análisis de Sportiche (1996), quien denomina Voces a las proyecciones correspondientes. 
La estructura resultante sugerida es, pues, la siguiente (debe tenerse en cuenta que el esquema representa la estructura inmediatamente anterior al desplazamiento del sujeto al especificador de la estructura temporal superior):

$$
\left[{ }_{\mathrm{VAc}} \text { clítico }\left[{ }_{\mathrm{S} v} \mathrm{SN} \text {-nominativo }\left[_{\mathrm{Sv}} v\left[_{\mathrm{SV}} \mathrm{V} \text { pro-acusativo }\right]\right]\right]\right]
$$

La posición del clítico en este análisis como elemento aparte del objeto propiamente dicho puede además considerarse equivalente a la de enfoques como los de Borer (1984), Suñer (1988), en que el clítico se comporta como un elemento funcional independiente, de modo afín a la categoría "Concordancia de Objeto" en Chomsky (1995). No existe, en todo caso, consenso en torno a la cuestión de la posición básica de los clíticos de objeto, que algunos autores (Uriagereka 1995; Torrego 1998; Roberts 2010) consideran parte de la composición interna del objeto, obligada ulteriormente a desplazarse. Nosotros nos decantamos por la primera opción, atendiendo a que resulta más acorde con el carácter no interpretable, propio de otros casos de concordancia, del clítico de objeto. Es decir, la relación propiamente semántica de complementación queda establecida a través del ensamble inicial, que da lugar al conjunto asimétrico [ ${ }_{\mathrm{SV}}$ V pro] (Chomsky 2008). Los niños con TEL no muestran dificultad en captar conceptualmente esta relación. Por el contrario, la relación entre el clítico y el complemento abstracto (es decir, $\left[{ }_{\mathrm{VAc}}\right.$ clítico $_{\mathrm{i}} \ldots$ pro $\left._{\mathrm{i}}\right]$ ) se establece a distancia y es puramente formal. Les plantea un problema para cuya resolución parecen estar menos capacitados, dando lugar a las discordancias comentadas, que no trascienden al plano semántico.

De acuerdo con el modelo "sonda-meta" que adoptamos, las metas potenciales de una sonda (en este caso el clítico) incorporan rasgos también no interpretables que actúan como guías en la operación de búsqueda. En nuestro caso, el SN sujeto, una de las potenciales metas, incorpora el rasgo "nominativo" y el 'pro' objeto el de "acusativo". En circunstancias normales, esto debería servir para que el clítico de objeto se salte en su búsqueda al SN-nominativo (el rasgo "nominativo" actúa como una especie de "inhibidor" de todo lo relacionado con los objetos) y concuerde en cambio con el pro-acusativo. Razones independientes a nuestro caso en particular dan pie a pensar que las gramáticas de los niños con TEL son idiosincráticas precisamente por el hecho de ser tendentes a manifestar una pobre fijación de rasgos² ${ }^{2}$ Vares (2017,

Esta y otras características del TEL tienen probablemente su origen en la limitada capacidad de memoria de trabajo de los niños que padecen el trastorno (Montgomery 2000, Vugs et al. 2006). Esto podría cuestionar la especificidad del trastorno y, también, la de la propuesta elaborada en este trabajo. Debe valorarse, sin embargo, que, para el minimalismo chomskiano, que en líneas generales asumimos en este trabajo, el componente nuclear del lenguaje es un sistema de computación, que, por definición, implica un dispositivo secuenciador y uno de memoria de trabajo. Que este último se 
2018) lo constata, por ejemplo, en el dominio de la flexión verbal o en el de la periferia izquierda en general (interrogación, fuerza ilocutiva, etc.). Pensamos, por esta razón, que en los ejemplos con el tipo de error del que nos estamos ocupando ocurre que los rasgos de caso no están presentes en sus portadores habituales. En consecuencia, la búsqueda por parte del clítico de una meta deja de estar guiada por aquellos rasgos. En ausencia de esta guía gramatical, el criterio de búsqueda pasa a ser otro alternativo en el que prevalece un criterio de economía (por tanto, un efecto de "tercer factor"): concretamente, el de concordar con la meta potencial más próxima, esto es, el SN sujeto. La Figura 4 ilustra, en términos de nuestro análisis, el contraste entre los casos típico (izquierda) y atípico (derecha).

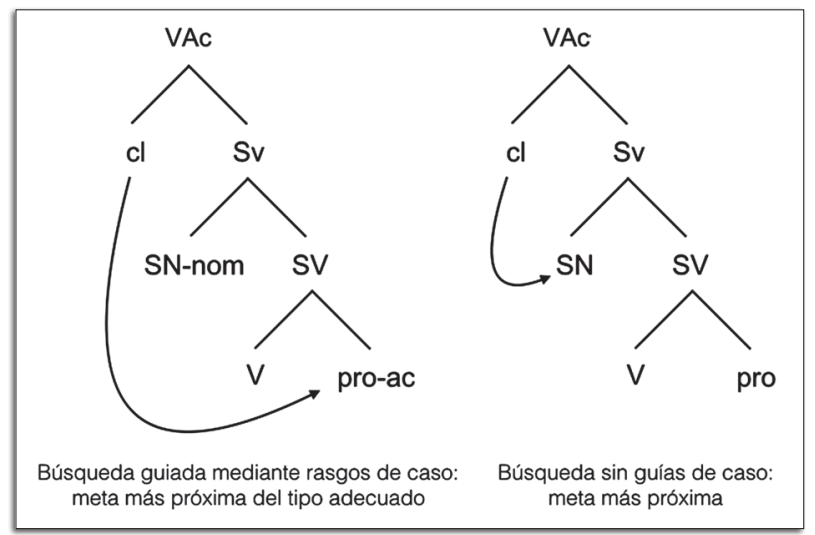

Figura 4. Validación de los rasgos del clítico, con

(izquierda) y sin (derecha) la asistencia de rasgos de caso.

Se trata de un efecto comparable al descrito por Grillo (2009) con relación a casos de agramatismo afásico, en los que la composición empobrecida de los sistemas de rasgos de algunos constituyentes determina una versión "extrema" de la condición de Minimidad (para otras aproximaciones afines en el caso de las afasias, véase Burchert et al. 2005; Grodzinsky 1990; Wenzlaff y Clahsen 2004). La versión estándar

encuentre afectado en los casos de TEL, como efectivamente creemos, no va contra la hipótesis que aquí sostenemos. Nuestra hipótesis es neutral acerca de si el dispositivo de memoria de trabajo es o no específico del lenguaje. Sospechamos que no lo es, es decir, que responde a una especialización lingüística que no conlleva necesariamente, sin embargo, especificidad lingüística. Con todo, si el daño afecta a la interfaz del dispositivo multipropósito de memoria con la secuenciación de expresiones lingüísticas, se sigue que pueden seguirse afectaciones única o predominantemente identificables en el área del lenguaje. Sobre estas cuestiones de arquitectura computacional, véase Balari y Lorenzo (2013). 
o "relativizada" de esta condición (Rizzi 1990) permite pasar por alto algún elemento mediador (en nuestro caso, el sujeto) entre los que deben ponerse en relación (en nuestro caso, el clítico y el objeto), gracias a que los rasgos de caso se encargan de marcar que el primero (el sujeto) es de un tipo diferente y no cuenta como el elemento más próximo capaz de entablar la relación. En ausencia de esos rasgos, el cómputo del elemento más próximo, como trata de ilustrar la Figura 4, resulta mucho más estricto o literal.

Nos interesa subrayar especialmente que rasgos como "nominativo" o "acusativo" pertenecen al tipo de rasgos que, de acuerdo con Chomsky, no realizan una aportación significativa a la oración, sino que forman parte del componente puramente gramatical de la facultad lingüística. En cambio, un principio de economía u optimización como "busca la meta más próxima" se encuadra entre los recursos de dominio general denominados como "tercer factor" (Chomsky 1995; 2005). Esto nos lleva a concluir que es el primer componente el que se encuentra sujeto a afectación en casos de TEL, lo que determina que los principios de optimización se apliquen de una manera mucho más "radical", al verse liberados del efecto constrictor de las condiciones específicamente gramaticales. Nótese que, de acuerdo con la propuesta, los rasgos puramente gramaticales resultan no ser los verdaderos instigadores de las operaciones en que se encuentran implicados, sino que desempeñan un papel más bien "coadjutor" en las mismas, balizando los puntos estructurales relevantes (véase, por ejemplo, Balari y Lorenzo 2018; Lorenzo 2018). La instigación propiamente dicha se debe a cuestiones interpretativas derivadas de los sistemas de pensamiento, que no parecen nuclearmente afectadas en cuadros como los estudiados ${ }^{3}$. Estos pueden ser más bien descritos, por tanto, como reflejo de un problema en el establecimiento de correspondencias entre el sustrato del pensamiento que instiga las operaciones y los dispositivos gramaticales que facilitan su correcta concreción en las formas de lenguaje sin afectación.

\section{MÁS PRUEBAS DE MINIMALISMO RADICAL: EFECTOS DE "ANTI-ARRASTRE" EN FORMACIÓN DE INTERROGATIVAS}

Esta sección trata de la formación de interrogativas y se centra en la tendencia observada entre niños con TEL de dejar in situ partes del sintagma interrogativo que típicamente son "arrastradas" conjuntamente con la palabra interrogativa. Se

De hecho, en la documentación de que disponemos, más allá de la obtenida a través de los Experimentos 1 y 2, no identificamos errores consistentes en la confusión del clítico correspondiente al argumento interno con el argumento externo (p. ej., * me bailo, * yo limpio yo, etc.), o la generalización del nominativo como caso por defecto. La cuestión parece, pues, determinada por el pensamiento expresado. Los errores se concentran en la réplica (no interpretable) de rasgos. 
trata de un fenómeno con una frecuencia de aparición no muy elevada, pero en todo caso significativamente persistente en las producciones de la población estudiada. La sección 3.1 explica el paradigma experimental que dio lugar a su observación, así como las características de la población y los resultados concretamente obtenidos. La sección 3.2 los interpreta, concluyendo, de nuevo, que se deben de la aplicación de un principio básico de economía, que se hace cargo de la conformación de la estructura interrogativa en ausencia de otros condicionantes gramaticales típicos.

\subsection{Participantes, materiales y resultados}

Identificamos tal fenómeno en una serie de test encaminados a evaluar la producción de oraciones interrogativas por parte de niños con TEL. Estos test consistían en tareas de elicitación, en las que se ofrecía a los niños la imagen de una chica emitiendo una frase (por ejemplo, debajo de la cama), conjuntamente con un personaje, presentado como su madre, que emitía un verbo (por ejemplo, encontrar) y una imagen sobrepuesta representando un estado de cosas relacionado (Figura 5). Lo que se pedía a los niños era que hiciesen una pregunta, sirviéndose del verbo asociado a la madre, para la cual la emisión de la niña pudiera ser una respuesta adecuada (por ejemplo, ¿dónde encontró el niño el coche?).

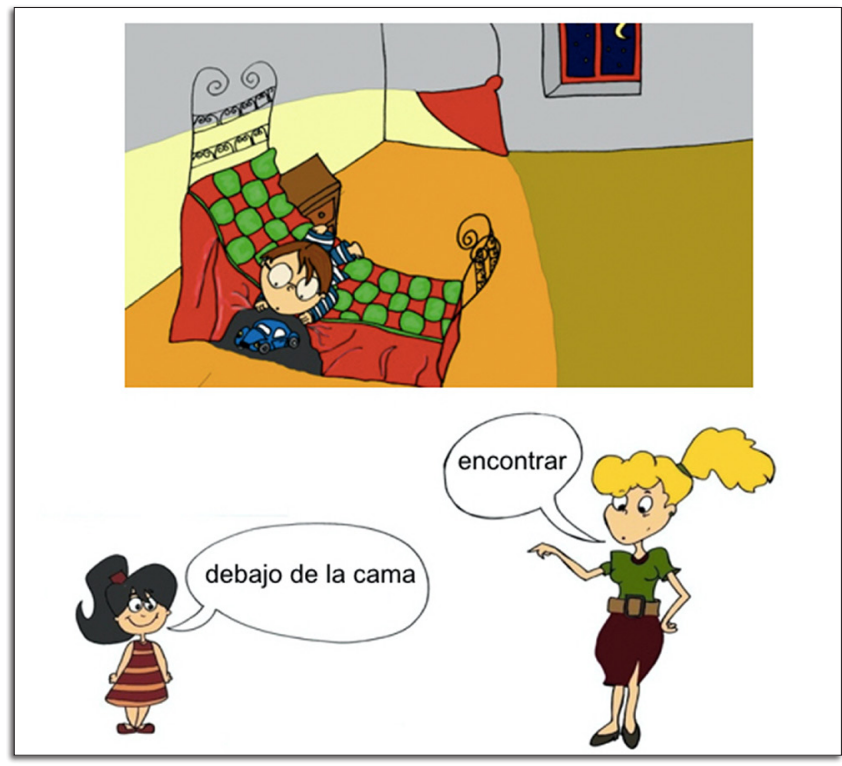

Figura 5. Ejemplo de imágenes y pistas de la prueba de elicitación de interrogativas. 
Con anterioridad a los test definitivos, los niños recibieron entrenamiento mediante dos diapositivas diferentes. En la primera, el examinador explicaba cuidadosamente a los niños lo que se esperaba de ellos y los animaba a realizar un primer intento. Si no lo lograban, se les mostraba la respuesta esperable. En la segunda, el examinador continuaba explicándoles la lógica de las pruebas y los animaba a nuevos intentos, esta vez sin proporcionar en ningún momento la respuesta. El experimento definitivo solo se iniciaba cuando los niños lograban producir respuestas esperables, incluso si contenían errores gramaticales. Verificamos que las pruebas eran comprensibles para los niños a partir de 10 años, después de que intentos previos con otras pruebas usadas en casos de agramatismo adulto (Martínez-Ferreiro 2010) resultasen ser de difícil comprensión en todas las edades consideradas. Realizaron las pruebas 12 niños con TEL (edad: 10;3 a 15;0, media: 12;7) y 12 niños con desarrollo típico (equiparados en edad). Los niños eran los mismos correspondientes a ese rango de edad del Experimento 2.

Aproximadamente la mitad de las producciones incorrectas de los niños con TEL contenía algún tipo de anomalía semántica o pragmática y casi una cuarta parte presentaba algún tipo de error gramatical, datos sin correspondencia en el caso de las producciones de los controles. Llamó especialmente nuestra atención que, a su vez, una tercera parte aproximadamente de esos errores gramaticales consistía en la aparición de una frase en la posición básica correspondiente a la palabra interrogativa, relacionada aquella semánticamente con esta (Tabla 4). Se trata de un fenómeno para el que encontramos equivalentes en casos estudiados por van der Lely y Battel (2003) con hablantes de inglés, y también por Gavruseva y Thornton (2001), aunque en el caso de niños de menor edad con desarrollo típico. Este tipo de error se ilustra en los ejemplos (9b) a (14b), que van precedidos de las producciones que esperábamos:

(9) a. ¿Qué come el niño?

b. ¿Quién come el niño? (N 10;8)

(10) a. ¿Adónde va el niño?

b. ¿Adónde va al colegio? (L 14;0)

(11) a. ¿Qué camiseta va a escoger el niño?

b. Ese niño, ¿qué va a escoger de camiseta? (I 14;11)

(12) a. ¿Qué encuentra el niño?

b. ¿Qué encuentra el niño un coche de color azul? (N 10;8)

(13) a. ¿Quién está sirviendo?

b. ¿Quién está sirviendo el camarero? (P 11;2) 
(14) a. ¿Quiénes van a ir?

b. ¿Quiénes van a ir la madre, el chico y el perro? (V 5;7)

\begin{tabular}{|c|c|c|}
\hline & Grupo TEL & Grupo control \\
\hline Correctas & $142(59 \%)$ & $238(99,17 \%)$ \\
\hline Completas & $99(41,4 \%)$ & $226(94,17 \%)$ \\
\hline Incompletas & $43(17,9 \%)$ & $12(5 \%)$ \\
\hline Incorrectas & $98(40,8 \%)$ & $2(0,8 \%)$ \\
\hline Error semántico/pragmático & $54(22,55 \%)$ & $1(0,42 \%)$ \\
\hline Error gramatical & $26(10,8 \%)$ & $1(0,42 \%)$ \\
\hline Oración afirmativa & $16(6,7 \%)$ & $0(0 \%)$ \\
\hline Ausencia de respuesta & $2(0,8 \%)$ & $0(0 \%)$ \\
\hline
\end{tabular}

Clave. - Completas: interrogativas con todos los constituyentes esperables. Incompletas: interrogativas con algún constituyente ausente, generando una cierta anomalía pragmática ${ }^{4}$. Error semántico/pragmático: interrogativas desviadas con relación al sentido o intención previstos (por ejemplo, con una palabra interrogativa inesperada). Error gramatical: orden de palabras inesperado (por ejemplo, palabra interrogativa in situ), elementos léxicos en la posición prevista para una huella, etc.

TABLA 4. Resultados de los test dirigidos a evaluar la producción de interrogativas.

\subsection{Análisis y discusión}

Es evidente, en primer lugar, que las oraciones que acabamos de ofrecer como ilustración son pragmáticamente atípicas, en relación con los correlatos esperados. Al construirlas, los niños parecen estar centrando con fuerza su atención en un elemento particularmente destacado de la situación (el niño, el colegio, la camiseta), e intentan incorporarlo en la interrogativa. El resultado es también semánticamente atípico, porque de ese modo la oración acaba por contener un elemento "restrictor", que restringe innecesariamente el rango de entidades de la clase a la que se aplica la palabra interrogativa: las oraciones $(9 \mathrm{~b})$ y (10b), por ejemplo, parecen significar anómalamente, según nuestra interpretación, “¿qué niño come?” o "¿a qué colegio va el niño?”, respectivamente. Los niños parecen, pues, estar generalizando anómalamente el uso de las llamadas interrogativas "ancladas al discurso" (Pesetsky 1987).

$4 \quad$ Estas oraciones responden en todos los casos a fenómenos regulares de omisión, que hemos constatado independientemente debidos a criterios de economía característicos de esta población de niños. Véase Vares (2016) y Lorenzo y Vares (en prensa). 
Sugerimos, atendiendo a lo anterior, que las oraciones comentadas contienen, en un primer momento, un constituyente que obedece a la estructura canónica "operador/ restrictor": es decir, [qu (x) / p (x)], donde "p" está en lugar de algún tipo de predicación; o sea, [qué (x) / niño (x)] en (6b), [dónde (x) / colegio (x)], en (7b) y [qué (x) / camiseta (x)], en (10b). En condiciones típicas, se desplazaría todo el constituyente (es decir, con "arrastre" del restrictor), para dar lugar a la estructura cuantificacional tripartita estándar: es decir, "operador/restrictor/alcance nuclear", donde el alcance nuclear consiste en el resto de la proposición temporalizada (Heim 1988; Partee 1995; Comorovski 1996); por ejemplo, [qué (x) / camiseta (x) / va_a_escoger (el niño, x)], para el caso de (11b).

Con este análisis como trasfondo, ocurre pues que en las construcciones atípicas de las que nos estamos ocupando se suma un error gramatical a los comentados arriba: concretamente, mientras que en condiciones normales el restrictor es arrastrado con la palabra/operador interrogativo (qué camiseta, en el caso de (11a)), permanece en cambio in situ en muchas producciones atípicas de niños con TEL (qué ... de camiseta, en el caso de (11b), donde interpretamos que la preposición actúa como una especie de aglutinador estructural, que entendemos hace innecesario el artículo en (9b) y (10b)).

De acuerdo con nuestra interpretación, en oraciones del tipo ilustrado de (9b) a (14b), los niños con TEL estarían aplicando un principio de economía como el originalmente postulado en Watanabe (1993):

(15) Economía de peso

El movimiento de material más pesado en más costoso (Watanabe 1993: 161; traducción de los autores).

En nuestro caso, interpretamos que la palabra interrogativa se desplaza como es habitual, pero sin el arrastre, también habitual, del restrictor, que permanece en cambio in situ y alivia así el peso del constituyente desplazado. Sugerimos, pues, un análisis como el de la Figura 6 (derecha), que contrastamos con el caso típico (izquierda). Algunos detalles del análisis se aclaran abajo: 


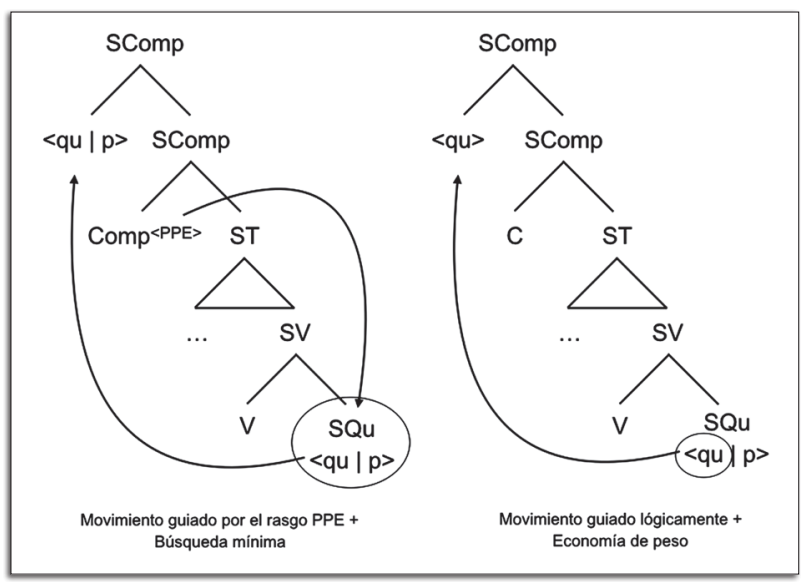

FIGURA 6. Movimiento de la palabra interrogativa con (izquierda) y $\sin$ (derecha) arrastre del restrictor.

En condiciones típicas, el movimiento del sintagma que contiene el operador interrogativo y el restrictor se explica como motivado, por una parte, por la presencia de un rasgo (PPE, por "principio de proyección extendido", en la terminología establecida desde Chomsky 1995), que fuerza tal operación. Por otra parte, Chomsky (2013) plantea que este rasgo identifica el candidato a ser desplazado manejando un criterio de "búsqueda mínima", en el sentido de que en cuanto encuentra una frase con el rasgo "qu" (SQu, en la figura), la arrastra en su conjunto, sin entrar en más consideraciones sobre su composición interna. Esto es lo que refleja la parte izquierda de la Figura 6, que se corresponde con la derivación típica de este tipo de construcciones (¿qué camiseta va a escoger el niño?). En condiciones de desarrollo atípico, en cambio, vuelve a ocurrir que el rasgo PPE, puramente gramatical, puede estar ausente de la derivación, debido al mismo tipo de efecto sugerido independiente por Grillo (2009) y Vares (2017). En tal circunstancia, entendemos que el movimiento de la palabra/operador interrogativo se efectúa por la motivación estrictamente semántica de generar una forma lógica acorde con la modalidad interrogativa, condicionada ahora por el principio general de economía reflejado en (15). Esto motiva que la palabra interrogativa se desplace al especificador de SComp, pero en este caso dejando atrás el restrictor, tal como se representa en la parte derecha de la Figura 6 (¿qué va a escoger el niño de camiseta?). Entendemos, por tanto, que se trata de un nuevo caso en que, en ausencia de un determinante gramatical (la condición impuesta por el rasgo PPE), la configuración de la estructura producida pasa a obedecer a criterios propios del "tercer factor": en este caso en particular, la presión de componer una forma 
lógica en que el operador tenga alcance sobre el resto de la estructura (imposición de los sistemas de pensamiento), más un principio general de economía que libera del desplazamiento requerido a tal efecto de otro material que no sea estrictamente el operador ${ }^{5}$. En contra de esta interpretación, se podría argumentar que, favoreciendo aún más la economía de la derivación, todo el sintagma debería quedar in situ, dando lugar a un formato de interrogación ampliamente constatado interlingüísticamente. No debe olvidarse, sin embargo, que la aparición de palabras y sintagmas interrogativos en esas lenguas está constreñida por los mismos criterios de localidad que en las lenguas con desplazamiento, lo que ha llevado a concluir que también en ellas se produce en último término el movimiento encubierto del operador o del sintagma que lo contiene (Huang 1998). Los niños con TEL estudiados dan respuesta de manera no problemática a la exigencia de tipo estructural que impone universalmente la forma lógica interrogativa. Se atienen además a la opción de hacerla visible en la forma fonológica, dando también así respuesta al parámetro al que responde el estímulo ambiental ("movimiento") en relación con esa propiedad estructural semantizada. Parecen satisfacerlo, no obstante, pasando por alto el aspecto estrictamente condicionado por un requisito formal ("arrastre"), dando lugar a la producción idiosincrática de palabras interrogativas desplazadas sin arrastre.

\section{DISCUSIÓN GENERAL: DESCRIBIR, EXPLICAR Y MÁS ALLÁ}

Tal como se planteó originalmente (Chomsky 1965: 18 y siguientes), el nivel de profundidad teórica habitualmente referido como "adecuación explicativa" servía para establecer el puente entre las teorías de la gramática y de la adquisición, de otro modo independientes entre sí. La distinción entre gramáticas "descriptiva” y

Cabe pensar en una estructura de partida coordinada del tipo: [come quién] \& [come el niño], donde ' $\&$ ' representaría un coordinador abstracto. La estructura iría seguida de una operación de focalización y otra de borrado de material idéntico, dando lugar a la forma final quién come el niño. En tal caso, tanto la estructura de partida como las operaciones subsiguientes podrían atenuar la complejidad propia de las construcciones interrogativas y hacerlas más accesibles a la población estudiada. Otra opción es que el operador interrogativo y el restrictor estén ocupando en estas construcciones su posición original, relacionándose de todos modos sin necesidad de movimiento. Aunque se trata de alternativas dignas de ser exploradas en profundidad, no podemos considerarlas acordes con lo que en general se aprecia en las producciones de los niños con TEL, que no manifiestan grandes dificultades en la generación de jerarquía estructural, lo que no favorece la alternativa basada en la coordinación, y sí, en cambio, en el establecimiento de relaciones internas entre los elementos jerarquizados, lo que a su vez no favorece la alternativa basada en la generación in situ más asociación sin movimiento. En apoyo de estas afirmaciones, véase, por ejemplo, Vares (2017), si bien con relación a la composición de relativas por parte de niños con TEL. 
"explicativamente" adecuadas se basaba en que un mismo lenguaje (técnicamente, un conjunto de secuencias o cadenas) podía ser alternativamente "descrito" a través de muchas gramáticas. El gramático debía por tanto seleccionar aquella que, entre todas ellas, fuese la que más verosímilmente explicase cómo se sobrepone el niño a la pobreza de los modelos del lenguaje en cuestión para adquirir un conocimiento pleno de la gramática subyacente (Berwick et al. 2011, Chomsky 2016). Las observaciones empíricas que hemos aportado en las secciones anteriores no se refieren al aprendiente "ideal" (en sentido técnico) del español, sino a aprendientes que se apartan del cronograma "típico" de adquisición y alcanzan por ello estados intermedios y estabilizados de competencia atípicos. Son, en todo caso, ejemplos de adquisición, que se relacionan igualmente con el desafío explicativo de la teoría lingüística bajo la concepción chomskiana del lenguaje y la lingüística. Tales casos plantean, efectivamente, el siguiente interrogante no trivial: ¿consiguen estos aprendientes sobreponerse a la pobreza del estímulo, si bien parcialmente, de un modo equivalente al del aprendiente "ideal"? ¿o deben, por el contrario, recurrir a estrategias de aprendizaje de un tipo cualitativamente distinto?

Sobre este trasfondo, el Supuesto de Continuidad (SC) se sigue naturalmente, puesto que la lógica que subyace a la distinción "descripción/explicación" es la de que el lingüista debe escoger una gramática para las producciones adultas de la comunidad de habla, bajo el supuesto de que es la que potencialmente facilita la explicación del proceso infantil de adquisición de la capacidad para realizarlas. Por esta razón, el enfoque puede razonablemente recibir la crítica de ser "adultocéntrico", aunque lo cierto es que, como ya comentamos en el arranque del trabajo, el verdadero supuesto es que las gramáticas adultas mantienen en su esencia el carácter infantil de los recursos que están en su base. Visto como un aspecto de la teoría de la adquisición, el SC concierne asimismo al estudio de los trastornos del desarrollo del lenguaje, en el sentido de que tiene relevancia preguntarse si la teorización sobre las gramáticas adultas también sirve de marco descriptivo y explicativo para comprender las desviaciones de las gramáticas de algún modo afectadas durante el desarrollo.

Desde una perspectiva descriptiva, en textos relevantes de lingüística clínica se asume implícitamente que los símbolos categoriales, las reglas de estructura de frase y transformacionales, así como el esquema general de organización arquitectónica de la gramática con que se estudian los estados de conocimiento lingüístico adultos, sirven igualmente para afrontar las descripciones de las gramáticas infantiles y de las gramáticas desarrolladas atípicamente. Nos podemos encontrar una formulación casi explícita de esta posición, por ejemplo, en Black y Chiat (2003: 7), donde se defiende que el estilo básico de descripción generativo-transformacional "servirá al especialista 
clínico para analizar las producciones de los niños y de los adultos con trastornos del lenguaje; los ayudará a identificar patrones de afectación, o de funcionamiento preservado, tanto en la producción como en la comprensión; y les facilitará la selección de estímulos relevantes y materiales para tareas de apoyo y actividades terapéuticas" (traducción de los autores).

Esta posición no es, sin embargo, totalmente consensual. Por ejemplo, una fuente tan autorizada como Crystal y sus colaboradores (1989: 34) se opone a ella, proponiendo en cambio que "las estructuras sintácticas que más directamente diferencian los tipos de habilidades que necesitamos caracterizar emergen como formatos a los que la GT [gramática transformacional] no presta atención" (traducción de los autores). En la misma fuente se concluye que "los rasgos distintivos más destacados [del lenguaje con afectaciones] son precisamente aquellos que no son directamente descriptibles en términos del modelo GT" (Crystal et al. 1989: 34; traducción de los autores). De todos modos, debe tenerse en cuenta que este texto no está exclusivamente dedicado a los trastornos lingüísticos del desarrollo, sino, sobre todo, a los sobrevenidos (como los debidos a traumatismos, accidentes vasculares o tumores), los cuales manifiestan habitualmente afectaciones mucho más devastadoras (véase, no obstante, Kerschensteiner y Huber 1975, donde la gramática transformacional se aplica a la afasia debida a problemas en el parto, así como Grodzinsky 1990, sobre la afasia en general). Por lo que se refiere a nuestra propia experiencia, que se ilustra en las secciones 2 y 3 de este trabajo, concluimos que, al menos en casos independientemente diagnosticados como TEL, los desarrollos más recientes de la gramática generativa transformacional pueden satisfacer plenamente necesidades de tipo tanto descriptivo como explicativo. En este sentido, no nos apartamos de líneas de investigación sobre el TEL tan influyentes como las hipótesis de la "etapa extendida del infinitivo opcional" (Rice et al. 1995), de la "complejidad computacional de la gramática" (van der Lely 2005), o del "déficit de la concordancia" (Clahsen et al. 1997), a todas las cuales subyace la idea de que el lenguaje con afectaciones es cualitativamente lenguaje típico, salvo por el efecto de un desfase en el cronograma de desarrollo o por ciertas afectaciones en aspectos reconocibles de las gramáticas típicas.

En el mismo marco de la teoría generativa del lenguaje, Chomsky (2004) ha propuesto una intrigante ampliación de la dicotomía original "adecuación descriptiva/ explicativa", a la que denomina "más allá de la adecuación explicativa" (en adelante, MAAE). El planteamiento de Chomsky es que la lingüística debe aspirar a un nuevo nivel de profundidad teórica, independiente, en su opinión, de la solución de los problemas del aprendizaje en contextos de pobreza de estímulo. Se trataría, en concreto, de explicar las propiedades de diseño del objeto adquirido, es decir, 
establecer el porqué de que el lenguaje muestre las características formales que de hecho exhibe. La tesis que específicamente respalda Chomsky al respecto es la de que el lenguaje se aproxima a lo que él entiende como un objeto "casi" perfecto, en el sentido de que se muestra altamente conforme a ciertos principios de diseño óptimo (economía/simplicidad, adaptación a los sistemas senso-motrices y de pensamiento con que se relaciona, eficiencia en el procesamiento de datos, leyes generales de la forma, principios y constricciones ineludibles del desarrollo orgánico, etc.), a todos los cuales se refiere conjuntamente, desde Chomsky (2005), como efectos de "tercer factor". Nótese que la apelación a tal factor diluye la distinción chomskiana original entre "competencia" y "actuación" lingüísticas (Chomsky 1965), y bloquea la posibilidad de juzgar el contraste entre las formas a que da lugar el desarrollo típico o atípico del lenguaje en términos de tales categorías. El llamado "tercer factor" reúne principios altamente generales y no específicos del lenguaje, que compiten con otros específicamente lingüísticos y altamente idiosincráticos, a los que conjuntamente denomina "gramaticales" y que remite a un genotipo lingüístico uniforme ("primer factor") o a la influencia diversificadora del ambiente ("segundo factor") (Chomsky 2005: 6). De acuerdo con Chomsky, la tesis minimalista más fuerte (Chomsky 2000) favorece las explicaciones basadas en terceros factores, los cuales apuntan a un diseño óptimo o perfecto, aunque la exploración empírica de la tesis parece conducir a la necesidad de postular un conjunto irreductible de rasgos o características gramaticales en todas las lenguas.

Uno de los principales motivos de este trabajo es el de proponer que la perspectiva MAAE abre la oportunidad de pensar acerca del SC también desde esta nueva perspectiva minimalista; es decir: ¿son las gramáticas infantiles y las gramáticas desarrolladas atípicamente continuas relativamente a las adultas en términos de diseño óptimo? En otras palabras: ¿se atienen las primeras a los mismos criterios de economía y eficiencia que las últimas? Si consideramos relevantes tales preguntas, entonces, de una manera no prevista por Chomsky, ocurre que la SC, que comúnmente solo relaciona con la teoría de la adquisición (es decir, con la perspectiva explicativa; Chomsky 2007; Hornstein et al. 2005), se relaciona también con las investigaciones minimalistas sobre las propiedades de diseño del lenguaje (es decir, con la perspectiva MAAE).

Chomsky plantea la tesis minimalista más fuerte mencionada más arriba como una pauta metodológica que debe llevar a cuestionarnos si un determinado aspecto del análisis lingüístico es reducible a un efecto de tercer factor o irreducible a cualquiera de ellos y, por tanto, específicamente gramatical. Aunque Chomsky se inclina a no vincular la tesis con la teoría de la adquisición (Chomsky 2007), nuestra propia visión es que lo anterior hace inevitable concluir que los aspectos del lenguaje sometidos 
a la pauta serán consecuencia de procesos de desarrollo espontáneo forzados por el factor identificado, en el primer caso, o de procesos guiados por intuiciones o conocimientos específicos sobre el lenguaje a disposición del niño con anterioridad a cualquier experiencia lingüística, en el segundo. Seguimos en este sentido propuestas como las de Longa y Lorenzo (2008) y Lorenzo y Longa (2009), o la tempranamente implementada en Radford (2000) (la visión más común, que desvincula el minimalismo de los problemas de la adquisición, se ilustra en cambio en Chomsky 2007, o Yang y Roeper 2011; para una síntesis, Hornstein et al. 2005). Desde nuestro propio punto de vista, la resolución de los problemas de la adquisición y de diseño van necesariamente de la mano. Son, en último término, uno y el mismo problema. La motivación última de esta posición nos parece ineludible: el Programa minimalista se basa en equiparar, en términos de poder causal sobre el diseño del lenguaje, el factor genético y los factores de optimización de las operaciones o de instanciación espontánea de las formas. Ambos tipos de factores condicionan el diseño alcanzado a través de su influencia sobre el proceso de desarrollo. El tercer (o terceros) factor(es), por tanto, participa(n) en la capacidad del organismo para sobreponerse a la pobreza de la estimulación en no menos medida que el primer factor. Su papel tiene tanto que ver, en consecuencia, con la perspectiva explicativa como con la que se sitúa más allá de la adecuación explicativa.

Lo que concretamente creemos haber mostrado en estas páginas es que las gramáticas infantiles con un desarrollo atípico son, con todo, gramáticas "típicas" desde el punto de vista del problema de diseño, es decir, desde una perspectiva que va "más allá de la adecuación explicativa", y continuas, en este sentido, tanto con las gramáticas infantiles con desarrollo típico como con las gramáticas adultas. Los aspectos de los que nos hemos ocupado concretamente se dejan explicar, de hecho, como debidos a la inestabilidad en las gramáticas atípicas de mecanismos específicamente gramaticales (rasgos de caso, el rasgo PPE, etc.), lo que da lugar a que efectos de tercer factor (minimidad, economía de peso, etc.) se hagan cargo de las áreas críticas correspondientes. En otras palabras, la consecuencia no son gramáticas imperfectas, sino, en el sentido del minimalismo chomskiano, gramáticas aún más perfectas que las gramáticas típicas.

\section{CONCLUSIÓN}

El Programa minimalista se centra en la cuestión de si el lenguaje es, o se aproxima a lo que podría considerarse un objeto perfecto. La respuesta parece ser que es un objeto "cercano" a la perfección, aunque no completamente perfecto. Para ser perfecto, de 
acuerdo con el estándar minimalista, el lenguaje debería ser capaz de funcionar sin la asistencia del tipo de mecanismos específicamente gramaticales que, sin embargo, están presentes en todas las lenguas. En este trabajo hemos defendido, por una parte, que las gramáticas con desarrollo atípico son continuas con relación a las gramáticas con desarrollo típico en un sentido profundo y acorde con los supuestos del minimalismo, puesto que las primeras también están sometidas a los criterios generales de optimidad y economía propios de las segundas, en tensión con imposiciones gramaticales de tipo más idiosincrático. Hemos defendido, además, que en este contexto de teorización sobre la forma de las gramáticas, las gramáticas con desarrollo atípico resultan no ser versiones empobrecidas de las gramáticas con desarrollo típico: son, muy al contrario, algo así como versiones enriquecidas, en que la tesis minimalista más fuerte se confirma más rotundamente. De acuerdo con esta conclusión, la gramática de los niños con TEL nos ofrece una ventana privilegiada para observar cómo sería el lenguaje si resultase ser más perfecto de lo que es. Bajo nuestra interpretación de los datos comentados, los niños con TEL son "minimalistas radicales". En todo caso, y siendo bien conscientes de los desafíos intelectuales que afrontan dada su condición, estas gramáticas parecen también mostrarnos que, en el caso del lenguaje al menos, ser algo menos que perfecto es ser lo suficientemente perfecto $^{6}$.

\section{AGRADECIMIENTOS}

Este trabajo ha sido realizado al amparo del proyecto de Investigación Desarrollo, Adquisición y Mecanismos de Variación Lingüística (DALiV; FFI2017-87699-P) (GL). Los autores desean expresar su agradecimiento a los dos revisores anónimos por sus valiosas sugerencias. Todos los errores que el texto pueda contener son de entera responsabilidad de los autores.

6 Esta conclusión puede resultar útil para realzar que la contraposición "perfecto / imperfecto" no obedece en el Programa minimalista a consideraciones valorativas. El Programa minimalista parte de la constatación de aspectos del diseño de las lenguas que parecen responder a una alta especificad de dominio, mientras que otros aparentan más bien ser abiertamente inespecíficos. Los primeros se consideran "imperfecciones" porque parecen presuponer una historia evolutiva particularizada para la función lingüística de la mente, de la que los segundos estarían, en cambio, dispensados. La razón de ser de las imperfecciones radica, pues, en la evolución de la especie y es difícil de descifrar, teniendo además en cuenta su aparente arbitrariedad funcional. No obstante, en el marco del paradigma biológico evo-devo (evolutivo del desarrollo), Balari y Lorenzo (2018) y Lorenzo (2018) han defendido recientemente que algunas de esas imperfecciones tienen un valor constructivo durante el desarrollo individual, que sería el responsable último de que hayan sido seleccionadas. La idea es consistente, argumentan, con el hecho de que en algunos casos de trastorno específico del desarrollo lingüístico esas características se vean característicamente afectadas. 


\section{NOTA}

Los autores se prestan a mostrar los materiales y documentación de este trabajo a cualquier investigador interesado. A tal fin, rogamos que contacten con ellos por vía electrónica.

\section{BIBLIOGRAFÍA}

ANDERSON, R. T. (2001). "Lexical morphology and verb use in child first language loss: A preliminary case study investigation". International Journal of Bilingualism, 5, 377-401. https://doi.org/10.1177/13670069010050040101

ANDERSON, R.T. (2003). "Language loss in Spanish-speaking children with and without SLI". Presentation at the Annual Convention of the American Speech-Language-Hearing Association, Chicago.

Balari, S. y Lorenzo, G. (2013). Computational Phenotypes. Towards an Evolutionary Developmental Biolinguistics. Oxford: Oxford University Press.

BALARI, S. y LoREnzo, G. (2018). "The internal, the external and the hybrid: The state of the art and a new characterization of language as a natural object. Glossa. A Journal of General Linguistics, 3(1), 22. https://doi.org.10.5334gjg1.330

BeDORE, L. M. y LEONARD, L. B. (2005). "Verb inflections and noun phrase morphology in the spontaneous speech of Spanish-speaking children with specific language impairment". Applied Psycholinguistics, 26, 195-225. https://doi.org/10.1017/ S0142716405050149

Berwick, R.C., Pietroski, P., Yankama, B. y Chomsky, N. (2011). "Poverty of the Stimulus Revisited”. Cognitive Science, 35, 1207-1242. https://doi.org/10.1111/ j.1551-6709.2011.01189.x

Black, M. y Chiat, S. (2003). Linguistics for Clinicians. London: Arnold.

Borer, H. (1984). Parametric Syntax. Case Studies in Semitic and Romance Languages. Dordrecht: Foris.

Burchert, F., Swoboda-Moll, M. y De Bleser, R. (2005). “Tense and Agreement dissociations in German agrammatic speakers: Underspecification vs. hierarchy. Brain and Language", 94, 88-199. https://doi.org/10.1016/j.band1.2004.12.006

Burzio, L. (1986). Italian Syntax. Dordrecht: Reidel. https://doi.org/10.1007/97894-009-4522-7

Chomsky, N. (1965). Aspect of the Theory of Syntax. Cambridge, MA: MIT Press. 
Chomsky, N. (1995). The Minimalist Program. Cambridge, MA: MIT Press.

Chomsky, N. (2000). "Minimalist inquiries: The framework", in R. Martin, D. Michaels y J. Uriagereka (eds.), Step by Step (pp. 69-126). Cambridge, MA: MIT Press.

Chomsky, N. (2001). "Derivation by Phase", in M. Kenstowicz (ed.), Ken Hale. A Life in Language (pp. 1-52). Cambridge, MA: MIT Press.

Сномsкy, N. (2004). "Beyond explanatory adequacy", in A. Belletti (ed.), The Cartography of Syntactic Structures. Vol.3. Structures and Beyond (pp. 104-131). Oxford: Oxford University Press.

Сномsкy, N. (2005). “Three factors in language design”. Linguistic Inquiry, 36, 1-22. https://doi.org/10.1162/0024389052993655

Chомsкy, N. (2007). “Approaching UG from below”, in U. Sauerland y H.-M. Gärtner (eds.), Interfaces + Recursion = Language $?$ Chomsky's Minimalism and the View from Syntax-Semantics (pp. 1-29). Berlin and New York: Mouton de Gruyter.

Chomsky, N. (2008). "On phases", in R. Freidin, C.P. Otero y M.L. Zubizarreta (eds.), Foundational Issues in Linguistic Theory: Essays in Honor of Jean-Roger Vergnaud (pp. 133-166). Cambridge, MA: The MIT Press. https://doi.org/10.7551/ mitpress/9780262062787.003.0007

Сномsкy, N. (2013). "Problems of projection". Lingua, 130, 33-49. https://doi.org/10.1016/j.lingua.2012.12.003

Сномsкy, N. (2016). "Poverty of stimulus: Unfinished business". Studies in Chinese Linguistics, 33, 3-16.

Clahsen, H. (1989). "The grammatical characterization of developmental dysphasia". Linguistics, 27, 897-920. https://doi.org/10.1515/ling.1989.27.5.897

Clahesen, H., BARTKE, S. y Göllner, S. (1997). "Formal features in impaired grammar: A comparison of English and German SLI children". Journal of Neurolinguistics, 10, 151-171. https://doi.org/10.1016/S0911-6044(97)00006-7

Comorovski, E. (1996). Interrogative Phrases and the Syntax-Semantics Interface. Dordrecht: Kluwer. https://doi.org/10.1007/978-94-015-8688-7

Crain, S. (2002). "The continuity assumption", in I. Lasser (Ed.), The Process of Language Acquisition (pp. 3-24). New York: Peter Lang.

Crystal, D., Fletcher, P. y Garman, M. (1989). Grammatical Analysis of Language Disability. 2nd Edition. London: Cole and Whurr. 
Gavruseva, E. y Thornton, R. (2001). "Getting it right: Acquisition of whose-questions in child English”. Language Acquisition, 9, 229-267. https://doi.org/10.1207/ S15327817LA0903_2

Gleitman, L.R. (1981). "Maturational determinants of language growth". Cognition, 10, 103-114. https://doi.org/10.1016/0010-0277(81)90032-9

GopNIK, M. y Crago, M.B. (1991). "Familial aggregation of a developmental language disorder”. Cognition, 39, 1-50. https://doi.org/10.1016/0010-0277(91)90058-C

GrILlo, N. (2009). “Generalized Minimality: Feature impoverishment and comprehension deficits in agrammatism”. Lingua, 119, 1426-1443. https://doi.org/10.1016/j. lingua.2008.04.003

Grodzinsky, Y. (1990). Theoretical Perspectives on Language Deficits. Cambridge, MA: MIT Press.

Gutiérrez-Clellen, V. F. y PeÑA, E. (2001). "Dynamic assessment of diverse children: A tutorial”. Language Speech and Hearing Services in Schools, 32, 212-224. https://doi.org/10.1044/0161-1461(2001/019)

Gutiérrez-Clellen, V. F., Restrepo, M. A. y Simón-Cereijido, G. (2006). “Evaluating the discriminant accuracy of a grammatical measure with Spanish-speaking children". Journal of Speech, Language, and Hearing Research, 49, 1209-1223. https://doi.org/10.1044/1092-4388(2006/087)

HeIm, I. (1988). The Semantics of Definite and Indefinite Noun Phrases. New York: Garland Publishers.

Hornstein, N., Nunes, J. y Grohmann, K.K. (2005). Understanding Minimalism. Cambridge: Cambridge University Press. https://doi.org/10.1017/CBO9780511840678

HuAng, C.-T. J. (1998). Logical Relations in Chinese and the Theory of Grammar. New York, Garland.

JACOBSON, P. F. y Schwartz, R. G. (2002). "Morphology in incipient bilingual Spanish-speaking preschool children with specific language impairment". Applied Psycholinguistics, 23, 23-41. https://doi.org/10.1017/S0142716402000024

Kerschensteiner, M. y Huber, W. (1975). "Grammatical impairment in developmental aphasia”. Cortex, 11 264-282. https://doi.org/10.1016/S0010-9452(75)80009-8

LEONARD, L.B. (1989). "Language learnability and specific language impairment in children”. Applied Psycholinguistics, 10, 179-202. https://doi.org/10.1017/ S0142716400008511 
Longa, V.M. y LoREnzo, G. (2008). "What about a (really) minimalist theory of language acquistion?". Linguistics. An Interdisciplinary Journal of the Language Sciences, 46, 541-570. https://doi.org/10.1515/LING.2008.018

Lorenzo, G. (2018). "Long-distance paradox and the hybrid nature of language". Biosemiotics, https://doi.org/10.1515/s12304-018-9331-1

Lorenzo, G. y LongA, V.M. (2009). "Beyond generative geneticism. Rethinking language evolution from a developmentalist point of view". Lingua. An International Journal of General Linguistics, 119, 1300-1315. https://doi.org/10.1016/j. lingua.2009.02.003

Lorenzo, G. y VAres, E. (2017). "The externalization component as the locus of specific impairments". Biolinguistics, 11, 1-19.

Lorenzo, G. y VAres, E. (en prensa). "The Distinctness Condition in SLI: More on the Specific Externalization Impairment. Glosema. Revista Asturiana de Llingüistica.

Macnamara, J. (1982). Names for Things. A Study of Human Learning. Cambridge, MA: MIT Press.

Martinez-Ferreiro, S. (2010). Towards a Characterization of Agrammatism in Ibero-Romance. Ph.D. Dissertation, Universitat Autònoma de Barcelona.

Mendoza Lara, E. (2016). Trastorno especifico del lenguaje. Avances en el estudio de un trastorno invisible. Madrid: Pirámide.

Michel, G.F. y Moore, C.L. (1995). Developmental Psychobiology. An Interdisciplinary Science. Cambridge, MA: MIT Press.

MONTGOMERY, J. W. (2000). "Verbal working memory in sentence comprehension in children with specific language impairment". Journal of Speech, Language, and Hearing Research, 43, 293-308. https://doi.org/10.1044/jslhr.4302.293

Morgan, G., Restrepo, M. A. y AuZA, A. (2009). "Variability in the grammatical profiles of Spanish-speaking children with specific language impairment". Hispanic child languages: Typical and impaired development, 50, 283.

Paradis, J., Crago, M. y Genesee, F. (2006). "Domain-general versus domain-specific accounts of specific language impairment: Evidence from bilingual children's acquisition of object pronouns". Language Acquisition, 13, 33-62. https://doi. org/10.1207/s153278171a1301_3

Partee, B.H. (1995). "Quantificational structures and compositionality”, in E. Bach, E. Jelinek, A. Kratzer y B.H. Partee (eds.), Quantification in Natural Languages (pp. 541-602). Dordrecht: Kluwer. 
Penner, Z., Schulz, P. y Wymann, K. (2003). "Learning the meaning of verbs: What distinguishes language-impaired from normally developing children?" Linguistics, 41, 289-319. https://doi.org/10.1515/ling.2003.010

Penner, Z., Wymann, K. y Schulz, P. (1999). "Specific Language Impairment revisited: Parallelism vs. Deviance. A learning-theoretical approach", in Z. Penner, Z., P. Schulz \& K. Wymann (eds.), Normal and Impaired Acquisition. Studies in Lexical, Syntactic, and Phonological Development II (pp. 1-26). Konstanz: Fachgruppe Sprachwissenschaft, University of Konstanz, Arbeitspapier 105.

Pesetsky, D. (1987). "Wh-in-Situ: Movement and unselective binding”, in E. Reuland y A. ter Meulen (eds.), The Representation of (In)Definitess (pp. 98-129). Cambridge, MA: MIT Press.

PINKER, S. (1984). Language Learnability and Language Development. Cambridge, MA: Harvard University Press.

RADFORD, A. (2000). "Children in search of perfection: Towards a minimalist model of acquisition". Essex Research Reports in Linguistics, 34, 57-74.

Restrepo, M. A., GutiérRez-Clellen, V. F. y Goldstein, B. (2004). "Grammatical impairments in Spanish-English bilingual children”, in B. Goldstein (Ed.) Bilingual language development and disorders in Spanish-English speakers (pp. 213-234). Baltimore, MD: P.H. Brooks.

Restrepo, M. A. y KRUth, K. (2000). "Grammatical characteristics of a Spanish-English bilingual child with specific language impairment". Communication Disorders Quarterly, 21, 66-76. https://doi.org/10.1177/152574010002100201

RiCE, M.L. y WeXLER, K. (1996). “Towards tense as a clinical marker of Specific Language Impairment in English-speaking children”. Journal of Speech, Language and Hearing Research, 39, 1239-1257. https://doi.org/10.1044/jshr.3906.1239

Rice, M.L., WeXler, K. y Cleave, P.L. (1995). "Specific Language Impairment as a period of extended optional infinite". Journal of Speech and Hearing Research, 38, 850-836. https://doi.org/10.1044/jshr.3804.850

Rizzi, L. (1990). Relativized Minimality. Cambridge: MIT Press.

Roberts, I. (2010). Agreement and Head Movement. Cambridge, MA: MIT Press. https://doi.org/10.7551/mitpress/9780262014304.001.0001

SAnZ-Torrent, M., BADIA, I., y Serra, M. (2007). "Contributions from bilingual Specific Language Impairment in Catalan and Spanish to the understanding of typical and pathological language acquisition”, in Pérez-Vidal, C., Juan-Garau, M. \& Bel, 
A. (eds.), A Portrait of the Young in the New Multilingual Spain (pp. 135-158). Clevendon: Multilingual Matters. https://doi.org/10.21832/9781847690241-010

Simon-Cereijido, G. y Gutierrez-Clellen, V. F. (2007). "Spontaneous language markers of Spanish language impairment". Applied Psycholinguistics, 28, 317339. https://doi.org/10.1017/S0142716407070166

Slobin, D.I. (1980). "The repeated path between transparency and opacity in language", in U. Bellugi, \& M. Studdert-Kennedy (eds.), Sign and Spoken Language: Biological Constraints on Linguistic Form (Dahlem-Konferenzen) (pp. 229-243). Weinheim: Verlag Chemie.

Sportiche, D. (1996). "Clitic constructions", in J. Rooryck y L. Zaring (eds.), Phase Structure and the Lexicon (213-276). Dordrecht: Kluwer. https://doi. org/10.1007/978-94-015-8617-7_9

SUÑER, M. (1988). "The role of agreement in clitic-doubling constructions". Natural Language and Linguistic Theory, 6, 391-434. https://doi.org/10.1007/BF00133904

Tomasello, M. (2000). “Do young children have adult syntactic competence?” Cognition, 74, 209-253. https://doi.org/10.1016/S0010-0277(99)00069-4

Torrego, E. (1998). The Dependencies of Objects. Cambridge, MA: MIT Press.

URIAGEREKA, J. (1995). "Aspects of the syntax of clitic placement in Western Romance". Linguistic Inquiry, 26, 79-123.

VAN DER LELY, H.K. (1998). "SLI in children: Movement, economy, and deficits in the computational system". Language Acquisition, 7, 161-192. https://doi. org/10.1207/s153278171a0702-4_4

VAN DER LELY, H.K. (2005). "Grammatical-SLI and the Computational Grammatical Complexity hypothesis". Frequences, 17, 13-20.

VAN DER LELY, H.K. y Battell, J. (2003). "Wh-movement in children with grammatical SLI: A test of the RDDR hypothesis". Language, 79, 153-181. https://doi. org/10.1353/lan.2003.0089

VAres, E. (2016). "Topic-drop residual en el Trastorno Específico del Lenguaje. Una interpretación desde la perspectiva del desarrollo". Revista Española de Lingüística, 46, 159-169.

VAres, E. (2017). Fenomenología lingüistica de los Trastornos Especificos del Lenguaje. Tesis Doctoral, Universidad de Oviedo.

VARES, E. (2018). Una aproximación devo-minimalista a los Trastornos Específicos del Lenguaje. Oviedo: EdiUno. 
Vugs, B., Knoors, H., Cuperus, J., Hendriks, M. y Verhoeven, L. (2016). “Interactions between working memory and language in young children with specific language impairment (SLI)". Child Neuropsychology, 22, 955-978. https://doi. org/10.1080/09297049.2015.1058348

Watanabe, A. (1993). Agr-Based Case Theory and Its Interaction with the A-Bar System. Ph.D. Dissertation, MIT.

WenzlafF, M. y Clahsen, H. (2004). "Tense and agreement in German agrammatism". Brain and Language, 89, 57-68. https://doi.org/10.1016/S0093934X(03)00298-0

YANG, C. y RoePer, T. (2011). "Minimalism and language acquisition", in C. Boeckx (ed.), Oxford Handbook of Linguistic Minimalism (pp. 551-573). Oxford: Oxford University Press. https://doi.org/10.1093/oxfordhb/9780199549368.013.0024 
